\title{
SOBRECARGA DO CUIDADO MATERNO À CRIANÇA COM CONDIÇÃO CRÔNICA*
}

Daniele Castro Barbosa ${ }^{1}$, Francisca Georgina Macedo de Sousa ${ }^{2}$, Andréa Cristina Oliveira Silva ${ }^{3}$, Ítalo Rodolfo Silva ${ }^{4}$, Thiago Privado da Silva ${ }^{4}$, Mirian Chaves Miranda Paiva ${ }^{5}$

RESUMO: Esta investigação objetivou classificar a sobrecarga do cuidado materno e verificar a relação desta sobrecarga com a condição crônica apresentada pela criança. Trata-se de estudo exploratório, transversal e descritivo apoiado em abordagem quantitativa. Os dados foram coletados entre agosto e outubro de 2010 por meio da Burden Interview - Escala de Sobrecarga para Cuidadores Informais e analisados por meio da estatística descritiva. Os resultados apontam predominância de níveis moderados de sobrecarga (86,7\%) decorrente do cuidado com o filho. A relação entre sobrecarga de cuidados e a condição crônica evidenciou maior sobrecarga em mães de crianças neuropatas. O conhecimento aqui desenvolvido poderá dar subsídios ao enfermeiro para o planejamento de suas ações e para atuação pautada na subjetividade do cuidado, possibilitando sensibilidade no lidar com a mãe cuidadora e a capacidade de vislumbrá-la enquanto receptora de cuidados. PALAVRAS-CHAVE: Enfermagem pediátrica; Saúde da criança; Doença crônica; Relações mãe-filho.

\section{OVERLOADING IN MATERNAL CARE FOR THE CHILD WITH A CHRONIC CONDITION}

ABSTRACT: This investigation aimed to classify the overload of maternal care and verify the relationship between this overload and the child's chronic condition. It is an exploratory, descriptive and cross-sectional study based in a quantitative approach. The data was collected between August and October 2010 using the Burden Interview - a scale for overload in informal carers - and was analyzed using descriptive statistics. The results indicate a predominance of moderate level of overload $(86.7 \%)$ resulting from care for the child. The relationship between being overburdened by care tasks and the chronic condition showed greater overburdening in mothers of children with neuropathies. The knowledge developed here can support the nurse in planning actions and for acting based in the subjectivity of the care, allowing greater sensitivity in dealing with the mother providing the care, and the capacity to see her as a receiver of care. KEYWORDS: Pediatric nursing; Children's health; Chronic illness; Mother-child relationships.

\section{SOBRECARGA DEL CUIDADO MATERNO AL NIÑO EN CONDICIÓN CRÓNICA}

RESUMEN: Esta investigación tuvo el objetivo de clasificar la sobrecarga del cuidado materno y verificar la relación de esta sobrecarga con la condición crónica presentada por el niño. Es un estudio exploratorio, transversal y descriptivo apoyado en abordaje cuantitativo. Los datos fueron recogidos entre agosto y octubre de 2010 por medio de la Burden Interview - Escala de Sobrecarga para Cuidadores Informales y analizados por medio de la estadística descriptiva. Los resultados apuntan predominancia de niveles moderados de sobrecarga (86,7\%) en consecuencia del cuidado con el hijo. La relación entre sobrecarga de cuidados y la condición crónica evidenció mayor sobrecarga en madres de niños neuropatas. El conocimiento desarrollado podrá dar subsidios al enfermero para el planeamiento de sus acciones y para actuación basada en la subjetividad del cuidado, posibilitando sensibilidad al lidiar con la madre cuidadora y la capacidad de mirarla como receptora de cuidados.

PALABRAS CLAVES: Enfermería pediátrica; Salud del niño; Enfermedad crónica; Relaciones madre-hijo.

*Este trabalho deriva da pesquisa "O cuidado materno e profissional à criança portadora de condição crônica" apoiado pela Fundação de Amparo a Pesquisa e ao Desenvolvimento Científico e Tecnológico do Maranhão e do Conselho Nacional de Desenvolvimento Científico e Tecnológico.

${ }^{1}$ Enfermeira. Mestranda pelo Programa de Pós-Graduação em Enfermagem da Universidade Federal do Maranhão - UFMA. Membro do Grupo de Estudo e Pesquisa na Saúde da Família, Criança e Adolescente - GEPSFCA. Bolsista Capes

${ }^{2}$ Enfermeira, Doutora em Enfermagem. Professora do Curso de Graduação e do Programa de Pós-Graduação em Enfermagem da UFMA. Coordenadora do Mestrado Acadêmico em Enfermagem e do GEPSFCA.

${ }^{3}$ Enfermeira Mestre em Saúde e Ambiente. Doutoranda em Enfermagem em Saúde Pública pela Universidade de São Paulo. Professora do Curso de Graduação em Enfermagem da UFMA. Membro do GEPSFCA.

${ }^{4}$ Enfermeiro. Mestrando pelo Programa de Pós-Graduação em Enfermagem da Escola de Enfermagem Anna Nery da Universidade Federal do Rio de Janeiro. Membro do Núcleo de Pesquisa Gestão em Saúde e Exercício Profissional da Enfermagem e do GEPSFCA. Bolsista Capes.

${ }^{5}$ Enfermeira Residente em UTI Geral pela UFMA. 


\section{INTRODUÇÃO}

As condições crônicas constituem o grupo de doenças que necessitam de cuidados contínuos e persistem por períodos longos, de vários anos ou décadas. Vistas sob esta perspectiva, as condições crônicas englobam uma vasta e diferenciada gama de agravos à saúde como: condições transmissíveis (diabetes, hipertensão, câncer), condições transmissíveis persistentes (HIV/Aids, hanseníase, tuberculose), distúrbios mentais e deficiências físicas ${ }^{(1)}$.

Atualmente, os avanços científicos e tecnológicos têm permitido o desenvolvimento de métodos diagnóstico e a implementação de terapêuticas adequadas, fato este que contribui sobremaneira para a identificação prévia de casos de condições crônicas. Em crianças têm-se percebido, nas últimas décadas, a queda na incidência de doenças agudas graves e o aumento na prevalência de condições crônicas. Associado a este fato está a redução de óbitos infantis, fator que contribui para incidências cada vez maiores de condições crônicas em crianças ${ }^{(2-3)}$.

Especificamente na infância, as condições crônicas carregam consigo certas particularidades que envolvem o sistema familiar como um todo. Nesse contexto, destaca-se que a família sente-se responsável pelos cuidados com a criança, tarefa esta complexa e por vezes dolorosa ${ }^{(4)}$. Aliado a esta realidade, está o fato de que são condições que exigem um nível de cuidados permanentes configurando-se, portanto, como fonte de sobrecarga para a família, sobretudo, para a mãe cuidadora, que geralmente está mais envolvida com o tratamento, acompanhando as internações e interagindo com os profissionais ${ }^{(5)}$.

Nesse processo, a mãe vivencia um cuidado diário e divide-se entre as responsabilidades de dona de casa e os cuidados à criança $\mathrm{e}$, em muitos casos, com as tarefas profissionais fora do lar destacando o papel múltiplo e particular que afeta as mães e a relação familiar $^{(6)}$. A mãe cuidadora, nessa dinâmica, também se depara com dificuldades que vão além das novas demandas de cuidados e perpassam pelo universo dos sentimentos. Ela vivência a angústia diante do diagnóstico do filho, convive com momentos de medo e incerteza e precisa assimilar a nova realidade imposta pela condição da criança $^{(7)}$.

Dessa forma, o cuidado materno à criança com condição crônica configura-se como uma tarefa exaustiva e geradora de sobrecarga devido às inúmeras necessidades advindas da condição da criança.
Essa sobrecarga, por sua vez, está relacionada não apenas às demandas de cuidado, mas também a sentimentos que acompanham a mãe constantemente como sentimentos de fragilidade, medo, preocupação, culpa bem como à administração da situação de desgaste vivenciada pela família ${ }^{(8)}$.

Partindo do pressuposto de que a sobrecarga de cuidados à criança com condição crônica incide principalmente sobre a figura materna, acarretando alterações intensas em sua vida e implicando na vivência de múltiplos sentimentos, delimitou-se como problema de pesquisa: quanto as mães se sentem sobrecarregadas com o cuidado ao filho com condição crônica e qual a relação entre o tipo de condição crônica da criança e a sobrecarga do cuidado materno?

A relevância desta investigação aponta para a necessidade de visualizar família, mãe e criança enquanto unidades de cuidados. O estudo possibilitará também a reflexão sobre a prática assistencial diária, ação necessária para que o enfermeiro repense suas atitudes de cuidado e seja, efetivamente, ator no desenvolvimento de estratégias de humanização e acolhimento $^{(9)}$. Sob esta perspectiva, objetiva-se com esta investigação classificar a sobrecarga do cuidado materno e verificar a relação desta sobrecarga com o tipo de condição crônica apresentada pela criança.

\section{MÉTODO}

Estudo exploratório, transversal e descritivo apoiado no método quantitativo de pesquisa. Participaram da investigação 59 mães e uma cuidadora (avó) de crianças com condições crônicas em tratamento nos cenários hospitalar e ambulatorial no período compreendido entre os meses de agosto e outubro de 2010. Entre as mães, duas possuíam dois filhos com a mesma patologia, totalizando assim, 62 crianças. Os cenários acima apontados foram escolhidos considerando-se a diversidade de ambientes onde se desenvolve o cuidado à criança com condição crônica.

Foi definido como critério de inclusão ser cuidadora de filhos com diagnóstico de doença que se insere como condição crônica. Os critérios de exclusão estavam relacionados à existência de problemas psiquiátricos e/ou auditivos da cuidadora que as impedissem de responder ao instrumento de coleta de dados.

O estudo foi desenvolvido inicialmente no serviço de internação pediátrica do Hospital Universitário 
Unidade Materno Infantil, no serviço ambulatorial da Clínica Escola Santa Edwiges da Associação de Pais e Amigos de Excepcionais. Diante da baixa rotatividade de crianças com condições crônicas nos serviços acima referidos optou-se por ampliar o local de estudo com a inclusão de casas de apoio a famílias de pacientes em tratamento no Hospital do Aparelho Locomotor. Todos esses serviços localizados em São Luís capital do Estado do Maranhão.

Utilizou-se como instrumento de coleta de dados a Burden Interview - Escala de Sobrecarga para Cuidadores Informais ${ }^{(10)}$. Essa escala é composta por 22 itens que devem ser respondidos utilizando uma escala Likert de cinco opções dispostas em progressão positiva. No último item o respondente é questionado sobre o quanto ele se sente sobrecarregado com a atribuição de cuidador. No presente caso, o quanto a mãe se sente sobrecarregada por conta dos cuidados com a criança. Portanto, a Burden Interview é uma escala que avalia, a partir da subjetividade do cuidador, o quanto este se sente sobrecarregado com o cuidado e no caso específico, a criança com condição crônica. Os escores de cada questão do instrumento são atribuídos pelo pesquisador, a partir de cada resposta do cuidador.

As respostas são pontuadas por escores que variam entre 0 e 4 com cinco possibilidades de resposta: Nunca (0), Raramente (1), Às vezes (2), Frequentemente (3) e Sempre (4). O último item do instrumento é marcado com escores de $0-4$ e as possíveis respostas: Nenhum pouco (0), Um pouco (1), Moderadamente (2), Muito (3) e Extremamente (4). O resultado da escala é computado através da soma dos escores, variando de 0 a 88 pontos, sendo que, quanto maior o escore maior a percepção de sobrecarga por parte do respondente. A partir da soma dos escores a sobrecarga é classificada em baixa sobrecarga com escores entre 04 e 20, sobrecarga moderada entre $21-50$, sobrecarga elevada escores entre $51-75$ e sobrecarga máxima entre $76-88$.

A investigação aqui descrita foi apreciada pelo Comitê de Ética do em Pesquisa do Hospital Universitário Unidade Presidente Dutra e aprovada sob parecer de número 192/10. Após esta etapa, iniciou-se a coleta de dados do estudo respeitando as determinações da Resolução 196/96 do Conselho Nacional de Saúde ${ }^{(11)}$. As participantes foram esclarecidas quanto aos objetivos e a metodologia da pesquisa, bem como foi solicitada a assinatura do Termo de Consentimento Livre e Esclarecido em duas vias. Buscou-se para a aplicação dos formulários a escolha de locais calmos e reservados, sem a participação de quaisquer outros membros da família. Após as orientações sobre seu preenchimento, a pesquisadora procedeu com a aplicação dos mesmos. Foram coletados dados adicionais relativos ao diagnóstico médico das crianças.

Os dados obtidos foram tabulados em planilha do Excel. Cada sujeito foi inserido na planilha com sua respectiva resposta para cada questão do instrumento. A partir da pontuação total de cada sujeito, foi possível classificá-los de acordo com os escores determinado pela Burden Interview. Os dados foram analisados por meio da estatística descritiva apresentando os resultados em frequências simples. Ao considerar o valor total dos escores de cada sujeito da pesquisa e para identificar aquele de maior frequência assim como a variabilidade entre os mesmos foi calculada a média e a moda.

\section{RESULTADOS}

Com relação às condições crônicas das crianças (Tabela 1) as mais frequentes foram as neuropatias com $63,1 \%$ seguidas por síndromes raras/erros inatos do metabolismo como as mucopolissacaridoses com $21 \%$. As nefropatias e as cardiopatias totalizaram, respectivamente, $9,7 \%$ e $8 \%$. Dentre as neuropatias destaca-se a paralisia cerebral decorrente de prematuridade, anóxia do parto e tumores cerebrais.

Tabela 1- Condição crônica específica por criança em acompanhamento ambulatorial e hospitalar segundo diagnóstico médico. São Luís, 2010

\begin{tabular}{lcc}
\hline Patologias & $\mathbf{n}$ & $\mathbf{\%}$ \\
\hline Neuropatias & 38 & 61,3 \\
Nefropatias & 06 & 9,7 \\
Cardiopatias & 05 & 8,0 \\
Síndromes raras/EIM & 13 & 21,0 \\
Total & 62 & 100,0 \\
\hline
\end{tabular}

Em relação à avaliação da sobrecarga do cuidado das participantes do estudo (Figura 1), 86,7\% das mães apresentaram sobrecarga moderada cujos escores variaram de 21 a 48, com média de 31,6 sendo 26 o escore mais frequente para esta classificação. Para a classificação baixa sobrecarga (10\%) os escores pontuaram entre 11 e 18 com média de 15,5 e 18 foi o escore mais frequente. Para mães classificadas com sobrecarga elevada $(3,2 \%)$ os escores variaram entre 53 e 55 com escore médio de 54 . A sobrecarga 
máxima não foi pontuada e não existiu sobrecarga zero, isto é, ausência de sobrecarga, uma vez que a soma dos escores totalizou no mínimo 11 pontos distanciando-se do escore mínimo proposto pelo instrumento que é quatro.

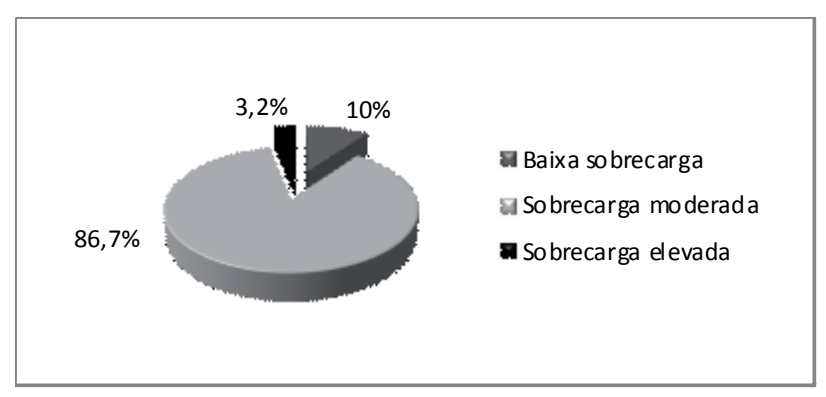

Figura 1 - Classificação da sobrecarga do cuidado de mães/ cuidadoras de crianças com condição crônica. São Luís, 2010

A figura 2 demonstra o padrão de resposta das mães entrevistadas para o último quesito da escala Burden Interview, que consiste em avaliar diretamente o quanto ela se percebe sobrecarregada por cuidar do filho. Para este quesito a resposta da mãe se diferencia das atribuídas anteriormente, como por exemplo, não existe a resposta Nunca e Raramente que para este quesito foram substituídas por Nenhum Pouco e Um Pouco. Assim, 30\% das mães não se sentem "Nem um Pouco" sobrecarregadas em cuidar do filho, outras 30\% sentem-se "Um Pouco" sobrecarregadas e 21,6\% sentem-se "Moderadamente sobrecarregadas. As respostas "Nem um pouco" e "Um Pouco" se contrapõem, a partir de uma compreensão subjetiva das mães, das opções "Nunca" e "Raramente" atribuídas às questões anteriores utilizadas para classificar a sobrecarga materna. As respostas "Muito" e "Extremamente" que indicam uma sobrecarga mais intensa, tiveram percentagens de respectivamente $15 \%$ e 3,4\%. Esse padrão de resposta demonstra resultados divergentes quando as mães são questionadas de forma direta e indireta sobre a

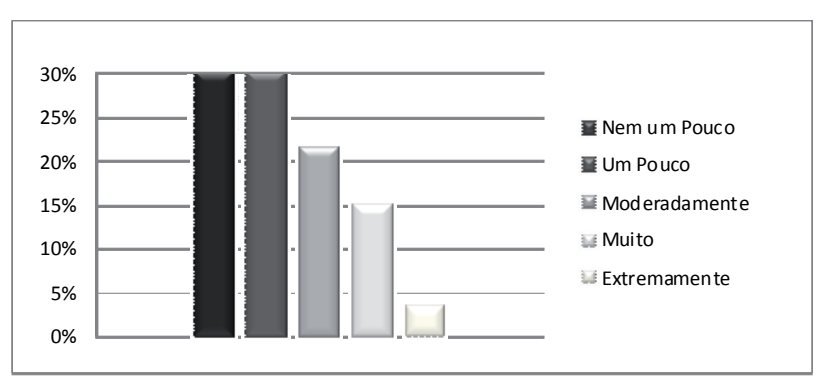

Figura 2 - Padrão de resposta de mães/cuidadoras de crianças com condição crônica segundo percepção de sobrecarga. São Luís, 2010

sobrecarga de cuidados.

Os resultados encontrados na relação entre a condição crônica da criança e a sobrecarga do cuidado materno (Tabela 2), apontam para maior sobrecarga entre as mães de crianças neuropatas, tendo em vista que 30 das 52 mães que compõem a classificação sobrecarga moderada possuem filhos com algum problema neurológico. O mesmo aconteceu com as duas únicas mães que apresentaram sobrecarga elevada. DISCUSSÃO

A literatura indica que as mães são as principais cuidadoras de filhos com condições crônicas e que elas estão expostas a uma maior sobrecarga do que os demais familiares. Nesse contexto, a sobrecarga pode ser definida como "problemas físicos, psicológicos ou emocionais, sociais e financeiros apresentados por cuidadores de pacientes dependentes"(12:39). Dessa forma, a sobrecarga do cuidado materno à criança com condição crônica está relacionada às consequências negativas resultantes da condição da criança ${ }^{(13)}$.

Cuidar de um filho com condição crônica se caracteriza pela aquisição de uma série de obrigações e estabelece uma relação intersubjetiva entre a díade mãe e filho. São obrigações dirigidas a resolver as necessidades gerais e específicas do filho e da condição crônica o que poderia caracterizar esse cuidado

Tabela 2- Sobrecarga do cuidado materno segundo grupo de condições crônicas. São Luís, 2010

\begin{tabular}{lccccc}
\hline $\begin{array}{c}\text { Condição } \\
\text { Crônica }\end{array}$ & Neuropatias & Nefropatias & Cardiopatias & $\begin{array}{c}\text { Síndromes raras/ } \\
\text { EIM }\end{array}$ & Total \\
Sobrecarga & $4(6,7 \%)$ & $1(1,6 \%)$ & $1(1,6 \%)$ & - & $6(9,9 \%)$ \\
\hline Baixa sobrecarga & $30(50,0 \%)$ & $5(8,3 \%)$ & $4(6,7 \%)$ & $13(21,7 \%)$ & $52(86,7 \%)$ \\
Sobrecarga moderada & $2(3,4 \%)$ & - & - & - & $2(3,4 \%)$ \\
Sobrecarga elevada & - & - & - & - & - \\
Sobrecarga máxima & $36(60,1 \%)$ & $6(9,9 \%)$ & $5(8,3 \%)$ & $13(21,7 \%)$ & $60(100 \%)$ \\
\multicolumn{1}{c}{ Total } & $36(60)$ & &
\end{tabular}


como de elevada sobrecarga. No entanto, os resultados apresentados nos faz compreender que o cuidado materno, encontra-se envolvido pela dimensão do amor e do afeto, portanto, se expressa pela dimensão subjetiva e pessoal, fato que pode ter caracterizado a maior frequência de mães com sobrecarga moderada. Mesmo aquelas com filhos neuropatas, que em virtude do agravo, apresentam maiores limitações físicas, motoras e cognitivas, e, consequentemente maior dependência de cuidados, não expressou para a mãe cuidadora elevada sobrecarga.

A condição crônica do filho atinge várias dimensões da vida da mãe cuidadora e se configura como sobrecarga porque requer que ela coloque, a si mesma, em segundo plano e adote um papel complementar àqueles já desempenhados ${ }^{(13)}$. Alguns fatores são responsáveis pelo aumento dessa sobrecarga de cuidados, tais como ausência de informação sobre a patologia e o tratamento, apoio inadequado dos profissionais e serviços de saúde, gravidade da doença, bem como, estratégias de enfrentamento inadequadas ${ }^{(6)}$. A dependência da criança, seu comportamento e o tempo que a mãe gasta para realizar os cuidados também se configuram como fatores que contribuem para uma maior ou menor sobrecarga ${ }^{(6)}$. Em contrapartida, a presença de suporte social fornecido por outros membros da família, pela rede social de amigos e ainda a capacidade sentida de poder controlar a situação podem atenuar a sobrecarga que incide sobre a figura materna ${ }^{(13)}$. No entanto, as demandas impostas pelas necessidades do filho no cotidiano das mães reforçam o seu papel de mãe e mulher no ambiente do lar e funcionam como extensão dos cuidados institucionais. A mãe compreende esse cuidado como dever, assume a luta diária em defesa do filho com amor e dedicação. Com esses sentimentos a mãe busca fazer tudo e a qualquer momento "para manter a criança o mais longe possível do perigo que a doença representa" ${ }^{\text {"(14:358) }}$ revelando o cuidado materno como protetor da vida da criança.

Além da sobrecarga de cuidados é comum as mães de crianças com condição crônica estarem suscetíveis à sobrecarga emocional, visto que elas vivenciam sentimentos como o sofrimento psicológico, o medo da morte da criança e a sensação de impotência diante da situação do filho ${ }^{(15)}$. Sob essa perspectiva, reforça-se a importância do suporte familiar como fator moderador da sobrecarga materna e destaca-se a convivência com outras mães em situações semelhantes como forma importante de apoio, tendo em vista, que elas poderão compartilhar sentimentos e experiências o que fornece segurança e auxílio no lidar com as incertezas geradas condição da criança ${ }^{(16)}$.

Assim, o padrão de resposta do estudo, que indica uma sobrecarga moderada, pode ser explicado pelo fato de que, para a mãe, realizar os cuidados para com o filho enfermo é uma experiência gratificante que se sobrepõe a qualquer prejuízo que possa surgir em consequência do cuidado à criança ${ }^{(17)}$. Outros autores compartilham da mesma idéia e afirmam que a vontade de cuidar, leva a mãe a adotar para si papéis nunca desempenhados, visando atender as necessidades do filho e melhorar sua qualidade de vida ${ }^{(15)}$. Portanto, essa motivação para o cuidado, é uma opção pessoal e está associada a sentimentos de amor e respeito, especialmente quando há um vínculo forte entre a mãe e a criança ${ }^{(15)}$.

No que diz respeito a relação entre a condição crônica da criança e a sobrecarga experimentada pela mãe cuidadora pode-se perceber que as crianças com neuropatias apresentam, em sua maioria, quadros graves que limitam a locomoção, a comunicação, a alimentação e até mesmo a capacidade respiratória, ficando todas essas funções sob responsabilidade da mãe e se configurando como fatores de aumento da sobrecarga. Aliado a esta realidade está o fato de que crianças com comprometimentos mais graves apresentam maior dependência e pouca participação na vida social $^{(17)}$, fatores estes contribuintes para uma maior sobrecarga em mães de crianças neuropatas.

\section{CONCLUSÃO}

Esta investigação evidenciou que as mães cuidadoras de crianças com condições crônicas apresentaram, em sua maioria, níveis moderados de sobrecarga de cuidado. Esse padrão, por sua vez, pode ser associado ao vínculo afetivo entre mãe e filho, o que torna o fato de cuidar da criança uma experiência gratificante, ancorada em sentimentos de amor e em atitudes de abnegação.

Nessa direção, pode-se inferir que por traz da condição da criança, há uma série de implicações que são vivenciadas pela família, pela própria criança e, em especial, pela mãe fato que representou para as autoras o desafio exigido pela investigação, no que diz respeito a sua dinâmica e temporalidade e suscitou muitos outros questionamentos, tais como: que necessidades emergem do processo de cuidado pela mãe cuidadora? Que fatores contribuem e fazem variar a sobrecarga materna? Como a família se organiza para diminuir a 
sobrecarga materna? Que sentimentos são vivenciados pela mãe e pela família ao cuidar do filho com condição crônica? São questionamentos que mobilizam e expressam necessidades para aprofundar conhecimentos acerca do processo de cuidado à criança com condição crônica e à família sua família. Compreender tais aspectos possibilitará ao enfermeiro o desenvolvimento de ações voltadas para a avaliação das relações afetivas, pessoais e familiares que se fazem presentes na vida da mãe cuidadora. Conhecer essas várias dimensões, portanto, permite a sensibilidade no lidar com a figura materna e a capacidade de vislumbrá-la enquanto receptora de cuidados. Dessa forma, o conhecimento aqui desenvolvido poderá dar subsídios ao enfermeiro para o planejamento consistente de suas ações e para uma atuação pautada na subjetividade do cuidado.

\section{REFERÊNCIAS}

1. Organização Mundial de Saúde. Cuidados inovadores para condições crônicas: componentes estruturais de ação: relatório mundial. Brasília: Organização Mundial de Saúde, 2003; 15.

2. Nóbrega RD. Collet N. Gomes IP, Holanda ER, Araújo YB. Criança em idade escolar hospitalizada: significados da condição crônica. Texto Contexto Enferm. 2010;19(3):425-33.

3. Wise PH. The future pediatrician: the challenge of chronic illness. J. pediatrics. 2007;151(5 Suppl):S6-10.

4. Nóbrega VM, Collet N, Silva KL, Coutinho SED. Rede e apoio social das famílias de crianças em condição crônica. Rev. Eletr. Enferm. [Internet] 2010;12(3). acesso em 13 dez 2011]. Disponível: http://www.fen. ufg.br/revista/v12/n3/v12n3a03.htm

5. Furtado MCC, Lima RAG. O cotidiano da família com filhos portadores de fibrose cística: subsídios para a enfermagem pediátrica. Rev. latino-Am. Enfermagem. 2003;11(1):66-73.

6. Rosset MOS. A sobrecarga na família de crianças com paralisia cerebral [dissertação]. Ribeirão Preto (SP): Universidade de São Paulo; 2009.

7. Almeida MI, Molina RCM, Vieira TMM, Higarashi IH, Marcon SS. O ser mãe de criança com doença crônica: realizando cuidados complexos. Esc. Anna Nery. 2006;10(1):36-46.

8. Marcon SS, Sassá AH, Soares NTI, Molina RCM.
Dificuldades e conflitos enfrentados pela família no cuidado cotidiano a uma criança com doença crônica. Cienc. cuid. saude. 2007;6(Suplem.2):411-9.

9. Mariano LRA, Backes DS, Ilha S, Nicola GDO, Freitas HMB, Ferreira CLL. Significados da internação hospitalar pediátrica na perspective de profissionais e familiares. Cogitare enferm. 2011;16(3):511-6.

10. Scazufca M. Brazilian version of the Burden Interview scale for the assessment of burden of care in carers of people with mental illnesses. Rev. Bras. Psiquiatr. 2002;24(1):12-7.

11. Ministério da Saúde (BR). Conselho Nacional de Sáude. Dispõe sobre diretrizes e normas regulamentadoras de pesquisa em seres humanos. Resolução n. 196/96 de 10 de outubro de 1996. Brasília; 1996.

12. Pontes AC. A maternidade de mães de crianças com doenças neurológicas crônicas: um estudo sobre a sobrecarga e qualidade de vida [tese]. Ribeirão Preto (SP): Universidade de São Paulo; 2008.

13. Bandeira M, Barroso SM. Sobrecarga das famílias de pacientes psiquiátricos. J. bras. psiquiatr. 2005;54(1):34-46.

14. Angelo M, Moreira PL, Rodrigues LMA. Incertezas diante do câncer infantil: compreendendo as necessidades da mãe. Esc. Anna Nery. 2010;14(2):301-8.

15. Silva CAM, Acker JIBV. O cuidado paliativo domiciliar sob a ótica de familiares responsáveis pela pessoa portadora de neoplasia. Rev. bras. enferm. 2007;60(2):150-4

16. Torres GV, Reis LA, Reis LA, Fernandes MH, Alves GS, Sampaio LS, et al. Funcionalidade familiar de idosos dependentes residentes em domicílio. Avaliação psicológica. 2009;8(3):415-23.

17. Camargos ACR, Lacerda TTB, Viana SO, Pinto LRA, Fonseca MLS. Avaliação da sobrecarga do cuidador de crianças com paralisia cerebral através da escala Burden Interview. Rev. Bras. Saude Mater. Infant. 2009;9(1):31-7. 